\title{
European legislation impedes critical care research and fails to protect patients' rights
}

Ronan MG Berg ${ }^{* 1}$, Kirsten Møller ${ }^{1,2}$ and Peter J Hancke Rosse ${ }^{3}$

\begin{abstract}
The European Clinical Trials Directive requires an informed consent from the patient or a proxy in drug trials. Although informed consent is a valuable tool to protect patients' rights in clinical trials, this requirement largely impedes research in critical care settings, and if pursued in this context, it does not provide the patient with adequate protection. Instead of insisting on informed consent, we suggest that the focus should be shifted towards two other ethically relevant elements in human experimentation: risk assessment and selection of research subjects. When reviewing protocols in which a waiver of consent is deemed necessary, the Ethical Review Board should ensure that non-therapeutic risks are minimal, that the research is specifically designed to benefit critically ill patients, and that it cannot be conducted under circumstances where an informed consent can be obtained. If the European Directive is changed accordingly, this permits clinical trials in critical care settings, while adequate protection from risky non-therapeutic procedures is ensured and exploitation of the patient as an easily accessible research subject is prevented.
\end{abstract}

The European Clinical Trials Directive includes a provision for informed consent from the patient or proxy consent from a legal representative in drug trials [1]. Although there is no doubt that informed consent is a valuable tool to protect patients' rights in clinical trials, it is only very rarely possible to obtain a valid informed consent in emergencies, where a life-saving intervention is required within seconds to minutes. If pursued, and the consent is obtained from either the patient or a proxy, it cannot be considered valid; because of the obviously

\footnotetext{
*Correspondence: ronan@dadlnet.dk

'Centre of Inflammation and Metabolism, Department of Infectious Diseases, Rigshospitalet, 2100 Copenhagen Ø, Denmark

Full list of author information is available at the end of the article
}

stressful situation, absence of duress cannot be presupposed, either with regard to a patient's or a relative's (proxy) consent. In contrast to the most recent revision of the Declaration of Helsinki [2], the European Directive offers no means of exemption from this requirement [1]. Thus, while the requirement of informed consent hampers clinical trials on potentially life-saving drugs in critical care settings [3], it does not protect the patient's rights in this context.

Informed consent is instituted in human experimentation in order to respect the autonomy of the research subject. Autonomy is a presupposition of agency and solely refers to agents who can understand and choose what they do [4]. Informed consent is not in itself an ethical principle, but an important procedure that permits human experimentation with the aim of benefiting society and future patients, without violating the basic rights of research subjects. However, since both medical treatment and, consequently, medical research also extend to those with incomplete autonomy, such as incapacitated critically ill patients, respect for autonomy cannot be considered the only or most important ethical principle. Other principles that express the basic rights of the research subject must be considered. In ethical terms, these basic rights are expressed in the 'categorical imperative,' which states that any human being possesses an inherent value and dignity and must be treated with respect, and therefore 'always at the same time as an end and never merely as a means to an end' [5]. To sustain this principle in subjects with incomplete autonomy that cannot consent to research, these subjects must be ensured adequate protection that reflects their vulnerability [6]. Instead of insisting on a prospective informed consent regardless of the context, it may therefore be worthwhile to accept the premise that informed consent or proxy consent cannot be obtained in all types of research. It is by no means a sine qua non, and comprises only one of three ethically relevant elements in human experimentation, the other two being risk assessment and selection of research subjects [6].

With regard to risk assessment, the accepted risks are tightly linked to the autonomy of the subject. Thus, higher risks are accepted when the subject is capable of 
providing a valid informed consent. In accordance with the principle of extra protection to patients with incomplete autonomy, risks must be minimised in incapacitated patients. These risks relate to both therapeutic and non-therapeutic procedures of the trial [6]. Therapeutic procedures are study interventions instituted with the intent of providing direct benefit to the patient. In drug trials, therapeutic procedures comprise the administration of drugs that have the prospect of benefiting the patient. These must pass the test of clinical equipoise, which signifies that the two interventions are roughly equivalent, in the sense that a genuine uncertainty concerning the comparable merits and disadvantages of each trial arm exists in the expert medical community [6]. The potential risks of these interventions are thus to be considered in light of their potential benefit for the individual patient. Non-therapeutic procedures are those interventions that are not instituted with therapeutic intent and are only intended to answer scientific questions of the study, such as additional laboratory tests and imaging to monitor treatment effects in detail. Since the patient cannot consent to these risks, these must be reduced to minimal risks [6]. Minimal risk implies that the probability and magnitude of harm or discomfort anticipated in the trial are not greater than those encountered by normal persons in daily life; a routine physical examination with blood sampling is considered a classical example of a procedure that involves a minimal risk [6]. Since the risks of the therapeutic procedures are unaffected by trial participation, the only additional risk imposed by inclusion in the trial is equivalent to that associated with a routine physical examination. As previously stated by Charles Weijer in Critical Care [7], such a risk assessment that distinguishes therapeutic from non-therapeutic components of the study shifts the focus from the potentially deleterious complications of critical illness per se to the actual risks imposed by participation in the study.

As for the selection of patients, the requirement of adequate protection entails that exploitation of these patients as easily accessible research subjects due to their impaired autonomy must be prevented. Thus, the overall aim of the research must be to specifically improve care of this patient group. Furthermore, it must be essential to the design and conclusions of the research that it is conducted under circumstances where it is by no means possible to obtain a valid informed consent [6].
If we want treatment of critically ill patients to be based on evidence-based principles, it is necessary to change the European Directive so that drug trials can be initiated under circumstances where it is impossible to obtain a valid informed consent from either the patient or a proxy. It is paramount that we ensure adequate protection of the individual patient in such studies, which is achieved by focusing on the scientific content, rather than the consent, which is neither sufficient nor necessary. The Ethical Review Boards should be equipped to determine whether participation in a trial is by any means contrary to the best interest of a given patient, by critically reviewing and monitoring study protocols in which a waiver of consent is deemed necessary. This permits clinical trials in critical care settings while ensuring that the individual patient is treated respectfully in accordance with the categorical imperative.

Competing interests

The authors declare that they have no competing interests.

Author contributions

RMGB drafted the manuscript. All authors made critical revisions and read and approved the final manuscript.

\section{Author details}

'Centre of Inflammation and Metabolism, Department of Infectious Diseases, Rigshospitalet, 2100 Copenhagen $\varnothing$, Denmark. ${ }^{2}$ Intensive Care Unit, Department of Anaesthesiology, Bispebjerg Hospital, 2400 Copenhagen NV, Denmark. ${ }^{3}$ Unit of Medical Philosophy and Clinical Theory, Institute of Public Health, University of Copenhagen, 1014 Copenhagen K, Denmark.

Published: 14 April 2011

References

1. European Parliament: Directive 2001/20/EC of the European Parliament and Council of 4th April 2001. Official Journal of the European Communities 2001, 121:33-44.

2. World Medical Association: Declaration of Helsinki. 59th WMA General Assembly: Seoul; 2008.

3. Robinson K, Andrews PJD: The European clinical trials directive and its impact on critical care and emergency research. Curr Opin Crit Care 2011, 17:141-145.

4. O'Neill O: Paternalism and partial autonomy. J Med Ethics 1984, 10:174-178.

5. Kant I: Grundlegung zur Metaphysik der Sitten. JF Hartknoch; 1785.

6. Weijer C: The ethical analysis of risk. J Law Med Ethics 2000, 28:344-361.

7. Weijer $C$ : The ethical analysis of risk in intensive care unit research. Crit Care 2004, 8:85-86

doi:10.1186/cc10113

Cite this article as: Berg RMG, et al.: European legislation impedes critical care research and fails to protect patients' rights. Critical Care 2011, 15:148. 